\title{
Black-box Modeling and Optimal Control of a Two-Phase Flow by Using Navier-Stokes Equations and Level Set Methods
}

\author{
A. Alessandri ${ }^{1}$, P. Bagnerini ${ }^{1}$, M. Gaggero ${ }^{2}$, L. Mantelli ${ }^{1}$, V. Santamaria ${ }^{1}$, A. Traverso ${ }^{1}$
}

\begin{abstract}
A modeling and control framework is proposed to describe the behavior of a water-ferrofluid two-phase 2D flow in the presence of a magnetic field and to devise proper optimal control actions. The dynamics of such a system descends from the cascade of magnetic field and Navier-Stokes equations. The former can be dealt with analytically, but this is not possible for the latter, which is usually treated numerically. The description of the motion of the interface between water and ferrofluid is accomplished by using level set methods. To overcome the computational difficulties when controlling such a system, a black-box model based on neural networks is constructed. Different kinds of neural networks are trained to account for the system behavior with an adequate precision in such a way to obtain a model that is well-suited for control. Optimal control is performed by using such black-box models with successful simulation results.
\end{abstract}

\section{INTRODUCTION}

This paper deals with modeling, identification, and control of a test rig composed by a water-ferrofluid two-phase flow in a thin squared tank under the effect of a magnetic field. The ferrofluid is a colloidal suspension based on light hydrocarbon carrier oils made up of single-domain magnetic particles immersed in a salt-saturated water mixture. The shape of the interface between liquid and ferrofluid is affected by the magnetic field generated through a grid of electromagnets placed under the tank. Owing to the tank thinness, it is possible to study the system in the two dimensional case. Level set methods have been adopted to describe the motion of the interface between water and ferrofluid, while the fluid dynamics is governed by Navier-Stokes equations. First, we have identified such a complex system by using a black-box model based on neural networks. Then, we have attacked the control of the shape of the ferrofluid by modifying the magnetic field generated by the electromagnets.

Level set methods are a popular tool to describe the evolution of interfaces in two-phase flows [1]. The interfaces are usually given by the zero level sets of the solution of Hamilton-Jacobi equations, i.e., a particular class of firstorder partial differential equations (PDEs). These methods can easily account for special kinds of behavior in shape dynamics that are not easily represented by other methods, like changes of topology. They find application in many

\footnotetext{
${ }^{1}$ A. Alessandri, P. Bagnerini, L. Mantelli, V. Santamaria, and A. Traverso are with the Department of Mechanical Engineering, University of Genoa, Via All'Opera Pia 15, I-16145 Genova, Italy (emails: alessandri@dime.unige.it, bagnerini@dime.unige.it, mantelli@dime.unige.it, vincenzo.alessandro.santamaria@gmail.com, alberto.traverso@unige.it).

${ }^{2}$ M. Gaggero is with the National Research Council of Italy, Via De Marini 6, I-16149 Genova, Italy (email: mauro.gaggero@cnr.it).

The authors gratefully acknowledge financial support from the AFOSR with grant FA9550-15-1-0530.
}

fields, such as physics, chemistry, computational fluid dynamics, and image processing [1]. Even if level set methods have been extensively studied to simulate the motion of interfaces, only few results are available about the possibility of controlling them by using some paradigm of optimal control [2]-[4].

The main difficulty when driving an interface between two fluids consists in the necessity of dealing with both the physics of the process (described by Navier-Stokes equations) and a suitable description of the interface (described by level set methods). Unfortunately, the resulting model based on the cascade of the physical setup based on Navier-Stokes equations and level sets turns out to be very computationally demanding if one wants to use it for the purpose of control. Thus, in this paper we construct a black-box approximate model of the cascade of Navier-Stokes and level set equations by using suitable nonlinear approximators. The goal is the fast computation of the control inputs in real time, without requiring the online numerical solution of the two PDEs. Such inputs are obtained by minimizing a performance index that depends on the shape of the interface.

Among the various choices for the family of nonlinear approximators, neural networks appear to be well-suited to our scope. This class of approximators includes onehidden-layer networks, which exhibit the powerful feature that consists in requiring a small number of parameters (i.e., the neural weights) to ensure a given approximation accuracy, especially in high-dimensional settings [5]. As it is well known, one-hidden-layer sigmoidal neural networks may guarantee uniform approximations with upper bounds depending on a number of parameters that grows at most polynomially with the dimension of the input of the function to be approximated (see, e.g., [6], [7], and the references therein). Based on the aforesaid, a number of applications are reported in the literature where the original problem is approximated through a learning process that is computationally demanding but made off line [8]-[10].

As compared with previous works [2], [3], the main novelty of this paper concerns the fact that here we deal with a cascade of two equations (i.e., the Navier-Stokes and the level set ones) instead of only controlling the HamiltonJacobi equation at the basis of the level set paradigm. To face the computational difficulties when dealing with a cascade of PDEs, here we propose the offline construction of an approximate black-box model based on neural networks.

Concerning optimal control, the choice of the control action that minimizes a given performance index is essentially in a finite set of possibilities. In fact, the electromagnetic 


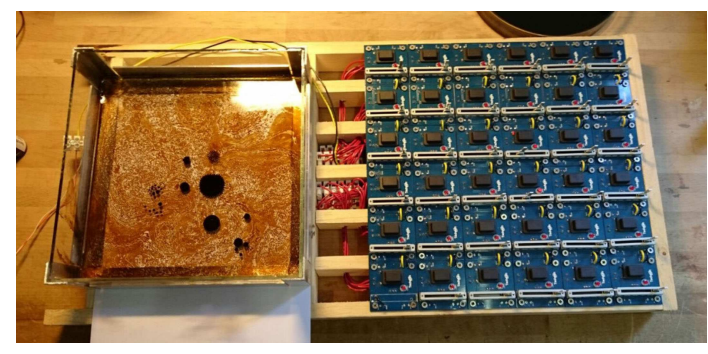

Fig. 1. Picture of the considered test rig.

field generated by the magnets derives from three admissible values for the current intensity, corresponding to the maximum of the intensity, half of it, and zero. Since the problem is of combinatorial nature, it cannot be solved by means of optimization methods that require the computation of the gradient or higher-order derivatives of the cost function. The use of nonderivative methods, such as the direct search ones, is mandatory [11]. Specifically, we will adopt a generalized pattern search (GPS) algorithm, which consists in performing a local search on a grid around the current point in such a way to reduce the cost at each iteration [12]. Simulation results show that such heuristic approach for control, combined with the black-box model of the system based on neural networks, is able to provide satisfying results as a tradeoff between accuracy and computational effort.

The paper is organized as follows. In Section II, the complete model of the setup is described. The identification of the model by using a black-box approach based on neural networks is presented in Section III. The optimization approach for the computation of control actions is described in Section IV. Simulation results are shown in Section V. Conclusions are drawn in Section VI.

\section{Dynamic Model of the System}

The test rig is composed of a tank of water and ferrofluid under the effect of a matrix of driving electromagnets. These electromagnets can change the magnetic field in order to modify the shape of the ferrofluid. A ferrofluid is a liquid in which ferromagnetic nanoparticles are suspended in a carrier liquid. Specifically, we focus on the case of a light polar mineral oil. The size of the particles prevents their attraction since the inter-magnetic forces are low with respect to the Van der Waals ones of the surfactant. The ferrofluid is mixed with a salt-saturated water solution that provides total separation between the two fluids. The overall density is almost equal to the density of the single components to avoid strong stratification. A wooden frame is used to keep the setup together with a glass vessel to house the fluid and the electronic package to drive the magnets. A picture of the used testbed is reported in Fig. 1.

The model is based on the following assumptions: (i) both fluids are in the liquid state, (ii) both fluids are incompressible, and (iii) both fluids are Newtonian, i.e., their viscosity is independent from the flow speed, and therefore it is possible to consider their viscosity constant if we assume a constant temperature. Under these hypotheses, the fluid dynamics can be represented by the following dimensionless incompressible 2D Navier-Stokes equations:

$$
\left\{\begin{aligned}
u_{t}+p_{x}= & -u u_{x}-v u_{y}+\frac{1}{R e}\left(u_{x x}+u_{y y}\right) \\
& +\frac{1}{F r^{2}} g_{X} \\
v_{t}+p_{y}= & -u v_{x}-v v_{y}+\frac{1}{R e}\left(v_{x x}+v_{y y}\right) \\
& +\frac{1}{F r^{2}} g_{Y} \\
u_{x}+v_{y}= & 0
\end{aligned}\right.
$$

where $u$ and $v$ are the $x$ and $y$ velocity components, respectively, $p$ is the pressure, $R e$ is the Reynolds number, $\mathrm{Fr}$ is the Froude number, and $g_{X}$ and $g_{Y}$ are the $x$ and $y$ acceleration field components, respectively.

The momentum equations (1a) and (1b) describe the time evolution of the velocity field $(u, v)$. The incompressibility condition (1c) is not a time-dependent equation, but an algebraic condition. If we consider $p$ as a Lagrange multiplier, it is possible to obtain a new form of the Navier-Stokes equations (1) that is easier to solve numerically [13]. Thus, we focus on the following equations instead of (1):

$$
\left\{\begin{aligned}
u_{t}+p_{x}= & -\left(u^{2}\right)_{x}-(u v)_{y}+\frac{1}{R e}\left(u_{x x}+u_{y y}\right) \\
& +\frac{1}{F r^{2}} g_{X} \\
v_{t}+p_{y}= & -\left(v^{2}\right)_{y}-(u v)_{x}+\frac{1}{R e}\left(v_{x x}+v_{y y}\right) \\
& +\frac{1}{F r^{2}} g_{Y}
\end{aligned}\right.
$$

The acceleration field $\boldsymbol{g}$ is the resultant of the acceleration field $\boldsymbol{g}_{m}$ caused by the magnetic force acting on the ferrofluid and of the acceleration field $\boldsymbol{g}_{t}$ caused by the interfacial tension. The acceleration $\boldsymbol{g}_{m}$ depends on the magnetic field $\boldsymbol{B}$ generated by 36 electromagnets that are arranged in a $6 \times 6$ grid. To compute the magnetic field generated by each electromagnet, we use the equations proposed in [14] and [15]. Then, the overall field can be obtained from the field generated by each magnet using the classical superposition principle. Let us collect in the vector $\boldsymbol{a}$ the current intensity of the various electromagnets. Thus, the overall magnetic field $\boldsymbol{B}$ is a function of the vector $\boldsymbol{a}$ as follows:

$$
\boldsymbol{B}=h(\boldsymbol{a})
$$

The reader interested in the expression of the function $h$ is referred to [14], [15].

To compute the force acting on each magnetic particle of the ferrofluid, we use the following equation [16]:

$$
\boldsymbol{F}_{m}=\nabla \boldsymbol{B} \cdot \mu
$$

where $\mu$ is the magnetic moment of each particle. From $\boldsymbol{F}_{m}$ it is possible to obtain an approximate value of $\boldsymbol{g}_{m}$. In fact, consider a small element of ferrofluid with volume $V_{e}$. Since the ferrofluid behaves like a homogeneous fluid [17], the force $\boldsymbol{F}$ acting on the entire element of ferrofluid is

$$
\boldsymbol{F}_{m, e}=n_{p} \boldsymbol{F}_{m}
$$


where $n_{p}$ is the number of magnetic particles in the element. The acceleration $\boldsymbol{g}_{m}$ is then computed as

$$
\boldsymbol{g}_{m}=\frac{\boldsymbol{F}_{m, e}}{\rho_{f} V_{e}}=\frac{n_{p} \boldsymbol{F}_{m}}{\rho_{f} V_{e}}=\frac{n_{p} \mu}{\rho_{f} V_{e}} \nabla \boldsymbol{B}
$$

where $\rho_{f}$ is the ferrofluid density.

The interfacial tension, caused by unbalanced attractive forces [18], acts on the particles of water and ferrofluid near the interface. Likewise in [1], the acceleration field $\boldsymbol{g}_{t}$ caused by the interfacial tension can be expressed as

$$
\boldsymbol{g}_{t}=\frac{1}{\rho} \sigma \kappa \delta(d) \boldsymbol{n}
$$

where $\rho$ is the fluid density, $\sigma$ is the interfacial tension coefficient, $\kappa$ is the curvature of the interface, $\delta(d)$ is the Dirac $\delta$ function of the distance from the interface, and $\boldsymbol{n}$ is the normal versor to the interface.

The evolution of the interface between liquid and ferrofluid is taken into account through the paradigm of level set methods. Thus, the interface is implicitly represented as the zero level set of a multidimensional function $\phi(\boldsymbol{x}(t), t)$, where $\boldsymbol{x}(t)$ is the position and $t$ is the time. The evolution of $\phi$ is determined by the following Hamilton-Jacobi equation:

$$
\phi_{t}+\nabla \phi(\boldsymbol{x}(t), t) \cdot \boldsymbol{x}^{\prime}(t)=0
$$

where the velocity field $\boldsymbol{x}^{\prime}(t)$ depends on the fluid dynamics of the system. Since the interface is the zero level set of $\phi$, the normal $\boldsymbol{n}$ can be obtained as

$$
\boldsymbol{n}=\frac{\phi}{|\nabla \phi|}
$$

Using the expression of the normal, we can compute the curvature $\kappa$ in (7) as

$$
\kappa=\nabla \cdot \boldsymbol{n}=\nabla \cdot \frac{\phi}{|\nabla \phi|}
$$

and the distance $d$ from the interface as

$$
d=\left|\frac{\phi}{|\nabla \phi|}\right| \text {. }
$$

Combining (10), (11), and (9), we get the expression for $\boldsymbol{g}_{t}$ as follows:

$$
\boldsymbol{g}_{t}=\frac{1}{\rho} \sigma \kappa(\phi) \delta\left(\frac{\phi}{|\nabla \phi|}\right) \frac{\phi}{|\nabla \phi|}
$$

\section{IDENTIFICATION OF THE DYNAMIC MODEL}

The dynamic model presented in the previous section allows one to describe the evolution in space and time of the interface between the water and the ferrofluid depending on the current intensity of the different electromagnets. However, it is very computational demanding, as it requires the numerical solution of two PDEs, i.e., the Navier-Stokes one (2) and the level set Hamilton-Jacobi one (8). Such a computational difficulty may be a severe issue if the model is used to generate optimal control actions. Indeed, in this case it has to be executed several times to evaluate the effectiveness of a given control input over the others. Clearly, this can undermine the feasibility of the computation of optimal control actions on line.

Motivated by the desire of reducing the required computational effort, in this section we propose a black-box approach based on neural networks to approximate the functional relationship between the vector $\boldsymbol{a}$, representing the current intensity of the electromagnets, and the evolution in time and space of the water-ferrofluid interface. More specifically, it is possible to write the following:

$$
\phi=f(\boldsymbol{a}, t)
$$

where $f$ is a function resulting from the application of the model (2)-(12). Unfortunately, the analytic expression of $f$ is unknown. The idea is to approximate off line the unknown function $f$ in (13) by using some nonlinear approximation techniques, in order to be able to generate the mapping $(\boldsymbol{a}, t) \mapsto \phi$ almost instantaneously. In particular, the following procedure can be adopted:

- solve off line equations (2)-(12) for many different values of $t$ and $\boldsymbol{a}$ and collect the corresponding pairs given by $\boldsymbol{a}, t$ and the function $\phi$;

- apply some learning method to approximate such pairs. More specifically, let us denote the different values of $t$ and $\boldsymbol{a}$ as $t^{(i)}$ and $\boldsymbol{a}^{(i)}$, respectively, for $i=1, \ldots, N$, where $N$ is the number of samples. Moreover, let $\phi^{(i)}, i=1, \ldots, N$, be the corresponding function $\phi$ in the 1.h.s. of (13).

In order to find an approximation of the function $f$ in (13), we constrain it to take on a certain fixed structure given by

$$
\phi=\gamma(\boldsymbol{a}, t, \boldsymbol{w})
$$

where $\gamma$ is a parametrized function depending on the vector of parameters $\boldsymbol{w} \in \mathbb{R}^{p}$. By tuning the values of such vector we can change the shape of the function $\gamma$. In our case, the goal is to search for the optimal parameters that yield a "good" interpolation of the pairs $\left(\tilde{\boldsymbol{a}}^{(i)}, \phi^{(i)}\right), i=1, \ldots, N$, where $\tilde{\boldsymbol{a}}^{(i)}:=\left(\boldsymbol{a}^{(i)}, t^{(i)}\right)$.

Among the various alternatives for the approximating function $\gamma$ in (14), we focus on one-hidden-layer feedforward neural networks with sigmoidal activation functions. This choice is motivated by the availability of a huge literature on their approximating capabilities and the presence of efficient, ad-hoc developed algorithms for the selection of the optimal parameters [5]. With this choice, in the case of scalar outputs, the function $\gamma$ has the following expression:

$$
\gamma(\tilde{\boldsymbol{a}}, \boldsymbol{w})=\sum_{i=1}^{\nu} c_{i} \sigma\left(\sum_{j=1}^{n} a_{i j} \tilde{a}_{j}+b_{i}\right)+c_{0}
$$

where $\nu$ is the number of neurons, $\tilde{a}_{j}$ is the $j$-th component of $\tilde{\boldsymbol{a}}, \sigma$ is a sigmoidal activation function, $c_{0}, c_{i}, b_{i} \in \mathbb{R}$, and $a_{i}:=\operatorname{col}\left(a_{i 1}, \ldots, a_{i n}\right) \in \mathbb{R}^{n}, i=1, \ldots, \nu$. The vector of parameters to be optimized is given by $\boldsymbol{w}:=$ $\operatorname{col}\left(a_{1}^{\top}, \ldots, a_{\nu}^{\top}, b_{1}, \ldots, b_{\nu}, c_{0}, \ldots, c_{\nu}\right)$. Their optimal value is usually found by solving the following optimization problem corresponding to a mean square error criterion:

$$
\min _{w \in \mathbb{R}^{p}} \sum_{i=1}^{N}\left(\phi^{(i)}-\gamma\left(\tilde{\boldsymbol{a}}^{(i)}, \boldsymbol{w}\right)\right)^{2} .
$$




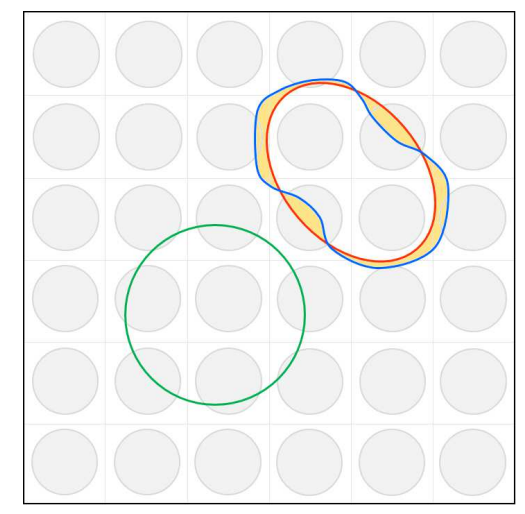

Fig. 2. Initial condition of the simulations used to train the neural networks (green), example of ferrofluid shape (blue), and example of reference shape (red). The symmetric difference between the ferrofluid shape and the reference shape is given by the yellow area. The grey circles show the positions of the electromagnets.

Concerning the theoretical properties of this kind of neural networks, it is known that they are endowed with the universal approximation property, i.e., they are able to approximate with arbitrary accuracy any "well-behaved" function. Moreover, they are particularly well-suited to dealing with high-dimensional problems, as the number of parameters that are required to obtain satisfactory approximations grows only polynomially with the dimension of the inputs. The interested reader is referred to, e.g., [6], [8] and the references therein for a deeper discussion.

The procedure to find the optimal values of the parameter vector $\boldsymbol{w}$ in (15) is called "training" in the neural network parlance, and a lot of efficient algorithms and corresponding software implementations exist in the literature, such as the classical backpropagation or Levenberg-Marquardt methods.

\section{Optimal CONTROL OF the INTERFACE}

Given a reference shape, our purpose is to find a configuration of the electromagnets such that the ferrofluid is shaped as desired when the system has reached the steady state. In other words, we want to find the optimal values of the vector $a$ containing the current intensity of the electromagnets so as to obtain desired shapes for the ferrofluid.

Toward this end, let us denote by $\Gamma^{d}$ a reference shape for the ferrofluid, and let $\Gamma(\boldsymbol{a})$ be the actual shape obtained at regime as the zero level set of the function $\phi$ that is the output of the neural network in (14). We adopt the symmetric difference ${ }^{1} \Gamma(\boldsymbol{a}) \Delta \Gamma^{d}$ as measure of the distance between $\Gamma(\boldsymbol{a})$ and $\Gamma^{d}$ (see Fig. 2). Thus, we have to solve the following optimization problem:

$$
\boldsymbol{a}^{\circ}=\underset{\boldsymbol{a} \in \mathbb{R}^{36}}{\operatorname{argmin}}\left(\Gamma(\boldsymbol{a}) \Delta \Gamma^{d}\right)
$$

At least in principle, problem (16) could be solved with any optimization routine. However, in this case the cost function does not change continuously with the input vector

${ }^{1}$ Given two sets $A$ and $B$, the symmetric difference is defined as $A \Delta B=$ $(A \cup B) \backslash(A \cap B)$. $a$. This is due to the spatial discretization used to solve equations (2)-(12). As a consequence, it is not possible to solve problem (16) using an optimization method that requires the computation of the gradient or higher-order derivatives of the cost function. Thus, the use of nonderivative methods, such as direct search ones, is mandatory [11]. Specifically, we will adopt a generalized pattern search (GPS) algorithm.

To explain the principle of the GPS method, we show how it can be used to minimize a generic cost function $F(x)$ with $x \in \mathbb{R}^{n}$. Let us consider $x_{k}$ at iteration $k$ of the GPS algorithm, together with mesh size $\Delta x_{k} \in \mathbb{R}^{+}$. We evaluate $F(x)$ in the points $x_{k}^{+}=x_{k} \pm \Delta x_{k} \cdot e_{i}, i \in\{1, \ldots, n\}$. The set of points $x_{k}^{+}$is called pattern. The set of vectors $e_{i}$ must be a spanning set of $\mathbb{R}^{n}$. We look for the $x_{k}^{+}$, such that $F\left(x_{k}^{+}\right)<$ $F\left(x_{k}\right)$, which gives the best result, i.e., the minimum value of $F\left(x_{k}^{+}\right)$. We denote such $x_{k}^{+}$by $x_{k}^{o}$. If we find this $x_{k}^{o}$, we set $x_{k+1}=x_{k}^{o}$ and $\Delta x_{k+1}=2 \Delta x_{k}$. Otherwise, if no $x_{k}^{o}$ is found, we set $x_{k+1}=x_{k}$ and $\Delta x_{k+1}=\Delta x_{k} / 2$. This iteration continues until $\Delta_{k}$ is smaller than a certain tolerance value [19]. Other stopping criteria may be adopted, such as the maximum number of performed iterations, the maximum number of cost function evaluations, and time limits.

\section{Simulation Results}

In this section, first we present the numerical results related to the construction of the black box model, and then we use it for the purpose of control.

\section{A. Construction of the Black-Box Model}

In order to train the neural networks as described in Section III, we must first collect the pairs $\left(\tilde{\boldsymbol{a}}^{(i)}, \phi^{(i)}\right)$ for $i=1, \ldots, N$. Toward this end, we solved 200 times the equations (2)-(12) starting from a certain initial condition and with different configurations of the electromagnets. Specifically, we considered the rectangle $[-3,3] \times[-3,3]$ as spatial domain. Concerning the initial condition, we assumed that the ferrofluid is in a circular shape, centered in $(-1,-1)$ with radius 1.1, as showed in Fig. 2. As regards the current intensity of the electromagnets, without loss of generality we assumed that such intensity is restricted to take on only three values, i.e., maximum one, half of the maximum, and zero.

The numerical solution of the Navier-Stokes equations (2) was performed in Matlab by suitably adapting the method proposed in [13] to account for the characteristics of the model, i.e., the presence of two fluids with different physical properties and the effect of a magnetic force acting on the ferrofluid and interfacial tension. The spatial discretization was done on a staggered grid, where $u$ and $v$ are placed on the vertical and horizontal cell sides, respectively, and $p$ is in the cell center. The discretization step was chosen equal to 0.1 . Concerning the time discretization, we selected a sampling time equal to 0.001 . Furthermore, we considered no-slip boundary condition on each wall of the tank for $u$ and $v$, as well as homogeneous Neumann boundary conditions for the pressure. As regards equation (8) modeling the evolution both in space and time of the interface between water and 
TABLE I

RESULTS OF THE NEURAL NETWORK TRAINING.

\begin{tabular}{lcc}
\hline & mean $\left(\Delta_{s}\right)$ & mean $\left(n_{c}\right)$ \\
\hline$\nu=5$ & 107.41 & 9.37 \\
$\nu=10$ & 93.78 & 6.17 \\
$\nu=15$ & 88.75 & 2.68 \\
$\nu=20$ & 86.51 & 2.02 \\
$\nu=25$ & 83.31 & 2.47 \\
$\nu=30$ & 82.93 & 1.38 \\
\hline
\end{tabular}

ferrofluid, we used the Matlab toolbox of level set methods developed by Mitchell [20].

The results of the 200 simulations described above were saved at 50 different time steps. Thus, we had at disposal a set of $N=10000$ pairs $\left(\tilde{\boldsymbol{a}}^{(i)}, \phi^{(i)}\right)$. Such pairs were then randomly divided into two subsets made up by 8700 and 1300 pairs, corresponding to the training set (i.e., the pairs used to find the optimal values of the parameter vector $\boldsymbol{w}$ as in (15)) and the test set (i.e., the pairs used to verify the effectiveness of the trained networks), respectively. Different neural networks were trained using the implementation of the Levenberg-Marquardt algorithm available in Matlab (function trainlm), corresponding to various numbers of neurons. Specifically, we varied $\nu$ from 5 to 30 .

The performances were evaluated by means of the following indexes, both computed over the 1300 pairs of the test set (the average values are reported in Table I):

- the symmetric difference $\Delta_{s}$ between the area occupied by the ferrofluid as obtained by solving (2)-(12) and the one provided by the neural network;

- the difference $n_{c}$ between the number of cells occupied by ferrofluid as obtained by solving (2)-(12) and the one provided by the neural network.

From the results reported in Table I, it turns out that the approximating capabilities of neural networks increase with the number $\nu$ of neurons, as expected. In fact, lower values for the average $\Delta_{s}$ can be observed is $\nu$ increases. Fig. 3 shows two examples of the interfaces between water and ferrofluid at certain randomly-extracted time steps as obtained by solving the system equations (2)-(12) and provided by the neural networks with $\nu=30$ neurons. One can notice that the two interfaces are similar, thus confirming the small value of the symmetric difference $\Delta_{s}$, i.e., the good approximating capabilities of the trained neural networks.

\section{B. Computation of Optimal Control Strategies}

In order to devise suitable control actions, we solved problem (16) by using the mesh adaptive GPS algorithm provided by the Matlab function patternsearch. More specifically, we adopted the neural network providing the best results in terms of accuracy, i.e., the network with $\nu=30$ neurons. The regime was supposed to be reached after $t=7$ ( $t$ is the dimensionless time used in the Navier-Stokes equations).

The results obtained by using the black-box model were compared with those provided by the numerical solution of equations (2)-(12) over a set made up of 12 different reference shapes. The results in terms of the symmetric difference $\Delta_{s}$ and the simulation time required to find a solution to problem (16) are showcased in Table II. Fig. 4 contains the results of the control for the reference shapes 5 and 8. Similar results have been obtained for the other shapes, but they are not reported for the sake of compactness. It turns out that the black-box model allows one to save a huge amount of time compared to the application of the full system equations (2)-(12), at the price of only a slight decay of performance. In fact, an average decay of about $16 \%$ of the values of $\Delta_{s}$ is experienced by using neural networks, but a saving of the $99 \%$ of computing time is achieved.

\section{CONCLUSIONS}

We have presented results on the black-box modeling and optimal control of a test rig made up by a square tank containing a two-phase fluid composed by water and ferrofluid and many electromagnets that allows one to change the shape of the ferrofluid through the resulting magnetic field. The dynamics of such a complex system is governed by Navier-Stokes equations together with a Hamilton-Jacobi equation describing the evolution of the interface according to the paradigm of level set methods. Both equations require suitable numerical approximations, which may be very computationally demanding in the case they are used to generate optimal control actions on line. Therefore, a blackbox model based on neural networks has been constructed to overcome such computational difficulties. Simulation results have confirmed the effectiveness of the proposed approach as a tradeoff between accuracy and required effort.

As a prospect of future work, we will investigate this approach on a larger set of test cases, and we will consider the effectiveness of other optimization methods different from the GPS algorithm. Moreover, we will collect experimental measurements directly from the test rig and use them to train new neural networks to be used for the control of the shape of the ferrofluid in the test rig.

\section{REFERENCES}

[1] J. Sethian, Level Set Methods and Fast Marching Methods, 2nd ed. Cambridge: Cambridge University Press, 1999, vol. 3.

[2] A. Alessandri, P. Bagnerini, and M. Gaggero, "Optimal control of level sets dynamics," in Proc. American Control Conf., 2014, pp. 22082213.

[3] A. Alessandri, P. Bagnerini, M. Gaggero, and A. Traverso, "Further results on the optimal control of fronts generated by level set methods," in Proc. American Control Conf., 2016, pp. 5225-5230.

[4] A. Alessandri, P. Bagnerini, and M. Gaggero, "Extended Kalman filtering to design optimal controllers of fronts generated by level set methods," in Proc. IEEE Conf. on Decision and Control, 2016, pp. 3966-3971.

[5] S. Haykin, Neural Networks, A Comprehensive Foundation. Singapore: Pearson Prentice Hall, 2005.

[6] A. R. Barron, "Universal approximation bounds for superpositions of a sigmoidal function," IEEE Trans. Inf. Theory, vol. 39, pp. 930-945, 1993.

[7] V. Kůrková and M. Sanguineti, "Comparison of worst-case errors in linear and neural network approximation," IEEE Trans. Inf. Theory, vol. 28, pp. 264-275, 2002.

[8] R. Zoppoli, M. Sanguineti, and T. Parisini, "Approximating networks and extended Ritz method for the solution of functional optimization problems," J. Optim. Theory Appl., vol. 112, no. 2, pp. 403-440, 2002. 

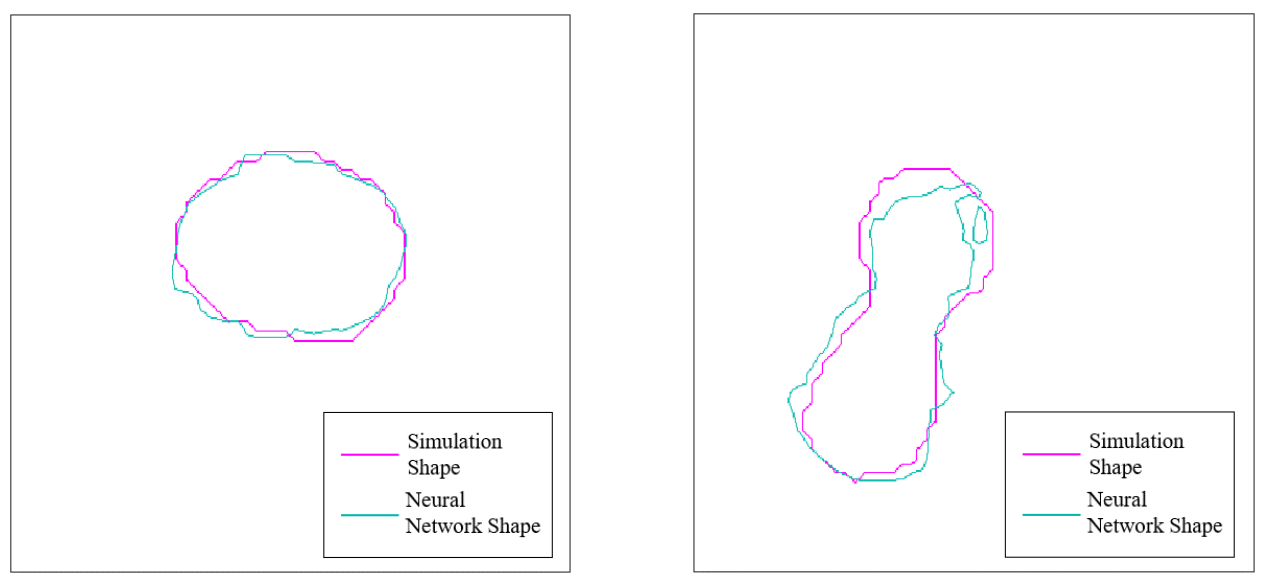

Fig. 3. Examples of interfaces between water and ferrofluid obtained by solving equations (2)-(12) and applying the neural network approximations.

TABLE II

RESULTS OF THE OPTIMAL CONTROL APPROACH.

\begin{tabular}{|c|c|c|c|c|c|c|c|c|c|c|c|c|}
\hline System model & \multicolumn{12}{|c|}{ Black-box model with $\nu=30$} \\
\hline Reference shape & 1 & 2 & 3 & 4 & 5 & 6 & 7 & 8 & 9 & 10 & 11 & 12 \\
\hline$\Delta_{s}$ & 94 & 242 & 236 & 189 & 169 & 121 & 94 & 86 & 111 & 248 & 217 & 158 \\
\hline Simulation time $[\mathrm{s}]$ & 590 & 668 & 800 & 600 & 622 & 648 & 616 & 623 & 640 & 628 & 640 & 631 \\
\hline System model & \multicolumn{12}{|c|}{ Full system equations (2)-(12) } \\
\hline Reference shape & 1 & 2 & 3 & 4 & 5 & 6 & 7 & 8 & 9 & 10 & 11 & 12 \\
\hline$\Delta_{s}$ & 242 & 170 & 165 & 66 & 202 & 94 & 140 & 71 & 95 & 276 & 67 & 54 \\
\hline Simulation time $[\mathrm{h}]$ & 34 & 31 & 32 & 24 & 21 & 26 & 19 & 36 & 26 & 19 & 22 & 35 \\
\hline
\end{tabular}
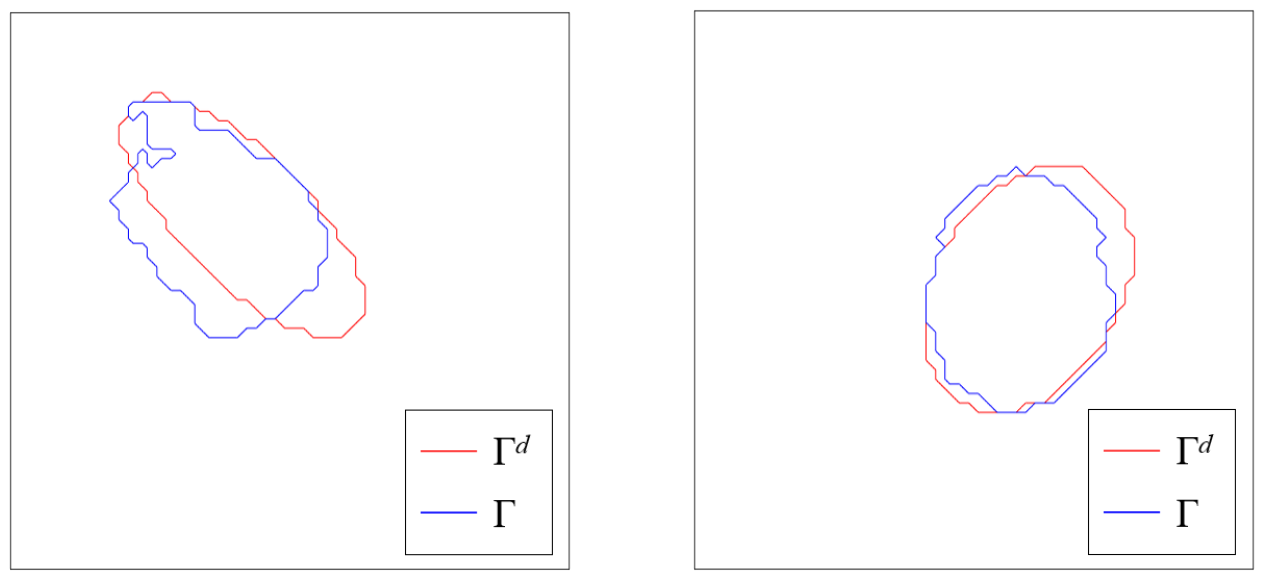

Fig. 4. Results of the control actions for the reference shapes 5 (left) and 8 (right).

[9] A. Alessandri, M. Gaggero, and R. Zoppoli, "Feedback optimal control of distributed parameter systems by using finite-dimensional approximation schemes," IEEE Trans. Neural Networks and Learning Systems, vol. 23, no. 6, pp. 984-996, 2012.

[10] M. Gaggero, G. Gnecco, and M. Sanguineti, "Approximate dynamic programming for stochastic N-stage optimization with application to optimal consumption under uncertainty," Computational Optimization and Applications, vol. 58, no. 1, pp. 31-85, 2014.

[11] D. P. Bertsekas, Nonlinear programming. Athena Scientific, 1999.

[12] C. Bogani, M. Gasparo, and A. Papini, "Generalized pattern search methods for a class of nonsmooth optimization problems with structure," Journal of Computational and Applied Mathematics, vol. 229, no. 1, pp. 283 - 293, 2009.

[13] B. Seibold, "A compact and fast Matlab code solving the incompressible Navier-Stokes equations on rectangular domains mit18086navierstokes.m," 2008.

[14] E. Callaghan, S. Maslen, and L. R. Center, The magnetic field of a finite solenoid, ser. NASA technical note, 1960. [Online]. Available: https://ntrs.nasa.gov/archive/nasa/casi.ntrs.nasa.gov/19980227402.pdf

[15] N. Derby and S. Olbert, "Cylindrical magnets and ideal solenoids," American Journal of Physics, vol. 78, no. 3, pp. 229-235, 2010.

[16] C. Scherer and A. M. Figueiredo Neto, "Ferrofluids: properties and applications," Brazilian Journal of Physics, vol. 35, no. 3A, pp. 718727, 2005.

[17] M. Petit, A. Kedous-Lebouc, Y. Avenas, M. Tawk, and E. Artega, "Calculation and analysis of local magnetic forces in ferrofluids," Przegld Elektrotechniczny, vol. 9B, 2011.

[18] P. Ghosh, Colloid and interface science. PHI Learning Pvt. Ltd., 2009.

[19] R. M. Lewis and V. Torczon, "Pattern search methods for linearly constrained minimization," SIAM J. Optim., vol. 10, no. 3, pp. 917941, 2000.

[20] I. Mitchell, "The flexible, extensible and efficient toolbox of level set methods," J. Sci. Comput., vol. 35, no. 2, pp. 300-329, 2008. 